\title{
Representation Theorems for Petri Nets
}

\author{
José Meseguer, Ugo Montanari, and Vladimiro Sassone
}

Dedicated to Prof. Wilfried Brauer on the occasion of his sixtieth birthday

\begin{abstract}
This paper retraces, collects, summarises, and mildly extends the contributions of the authors — both together and individually — on the theme of representing the space of computations of Petri nets in its mathematical essence.
\end{abstract}

\section{Introduction}

Among the semantics proposed for Petri nets [10] (see also [11, 13]), a relevant role is played by the various notions of process, e.g. $[\mathbf{1 2}, \mathbf{5}, \mathbf{1}]$, whose merit is to provide a faithful account of computations involving many different transitions and of the causal connections between the events occurring in computations. Bare process models, however, fail to bring to the foreground the algebraic structure of the space of computations of a net. Our interest, instead, resides on abstract models that capture the mathematical essence of such spaces, possibly axiomatically, roughly in the same way as a prime algebraic domain (or, equivalently, a prime event structure) models the computations of a safe net [9]. The research detailed in $[6,3,4,14,7,8,16]$ identifies such structures as symmetric monoidal categories — where objects are states, i.e., multisets of tokens, arrows are processes, and the tensor product and the arrow composition model, respectively, the operations of parallel and sequential composition of processes.

At a higher level of abstraction, the next important question concerns the global structure of the collection of such spaces, i.e., the axiomatisation 'in the large' of net computations. In other words, the space of the spaces of computations of Petri nets. Building on $[3,4,16]$, the work presented in $[15,17]$ shows that the so-called symmetric Petri categories, a class of symmetric strict monoidal categories with free (noncommutative) monoids of objects, provide one such an axiomatisation.

In this paper, we retrace and illustrate the main results achieved so far along these lines of research by the authors, both in joint work and individually. Also, we give a new presentation of the results of [15], already hinted at in [17], but never spelled out in detail before. Due to space limitations, we shall omit any discussion on related work in the literature.

1991 Mathematics Subject Classification. Primary 68Q55, 68Q10, 68Q05.

Key words and phrases. Semantics of Concurrency, Noninterleaving, Processes, Petri Nets. 


\section{Petri Nets as Monoids}

The idea of looking at nets as algebraic structures, e.g. $[\mathbf{1 3}, \mathbf{9}, \mathbf{1 8}, \mathbf{2}]$, has been given an original interpretation in [6], where nets are viewed essentially as internal graphs in categories of sets with structure, and monoidal categories are first used as a suitable semantic framework for them. Concerning the algebraic representation of net computations, along the lines described in the introduction, the following is the main result of $[6]$.

THEOREM. The commutative processes (see [1]) of a net $N$ are the arrows of $\mathcal{T}(N)$, the free strictly symmetric strict monoidal category on $N$.

Observe that, as a free construction, $\mathcal{T}(N)$ provides an axiomatisation of the commutative processes of $N$ as an algebraic theory, and thus moves a step towards a unification of the process and the algebraic view of net computations. As already mentioned, the algebra here consists of the operations of a monoidal category: tensor product and the arrow composition representing, respectively, parallel and sequential composition of processes.

Commutative processes, however, are somehow marginal in net theory. The next step was therefore to extend the result to the nonsequential processes of $N$ [5], the currently best-established notion of noninterleaving computation for nets.

\section{Concatenable Processes}

Concatenable processes of Petri nets have been introduced in $[3,4]$ to account, as their name indicates, for the issue of process sequential concatenation. The starting observation of loc. cit. is that such an operation has to do with merging tokens, i.e., instances of places, rather than places. In fact, due to the ambiguity introduced by multiple instance of places (multiple tokens in places), two processes of $N$ can be composed sequentially in many ways, each of which gives a possibly different process of $N$.

Therefore, any attempt to structure processes of $N$ as an algebra which includes sequential composition must disambiguate each token in a process. This is exactly the idea of concatenable processes, which are simply nonsequential processes in which, when needed, instances of places (tokens) are distinguished by appropriate decorations. This immediately yields an operation of concatenation: the ambiguity about multiple tokens is resolved using such additional information. The main result of $[\mathbf{3}, \mathbf{4}]$ is an axiomatisation of such a category, stated here in the improved enunciation proved in $[14,16]$.

THEOREM. The concatenable processes of a net $N$ are the arrows of $\mathcal{P}(N)$, obtained from the free symmetric strict monoidal category $\mathcal{F}(N)$ on $N$ by imposing the axioms

$$
\begin{aligned}
c_{a, b} & =i d_{a \otimes b}, \quad \text { if } a \text { and } b \text { are different places of } N, \\
s ; t ; s^{\prime} & =t, \quad \text { if } t \text { is a transition of } N \text { and } s \text { and } s^{\prime} \text { are symmetries of } \mathcal{F}(N),
\end{aligned}
$$

where $c, i d, \otimes$, and _; are, respectively, the symmetry isomorphism, the identities, the tensor product, and the composition of $\mathcal{F}(N)$.

This result matches the one for commutative processes in describing net behaviours as algebras in terms of universal constructions. Here, of course, a more complex algebraic structure - namely, symmetries and the related axioms above — is needed. 


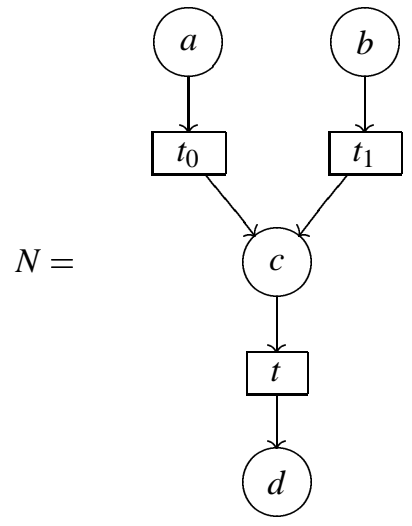

Places: $S_{N}=\{a, b, c, d\}$

Transitions: $\quad T_{N}=\left\{t_{0}: a \rightarrow c\right.$,

$$
t_{1}: b \rightarrow c
$$

$t: c \rightarrow d\}$

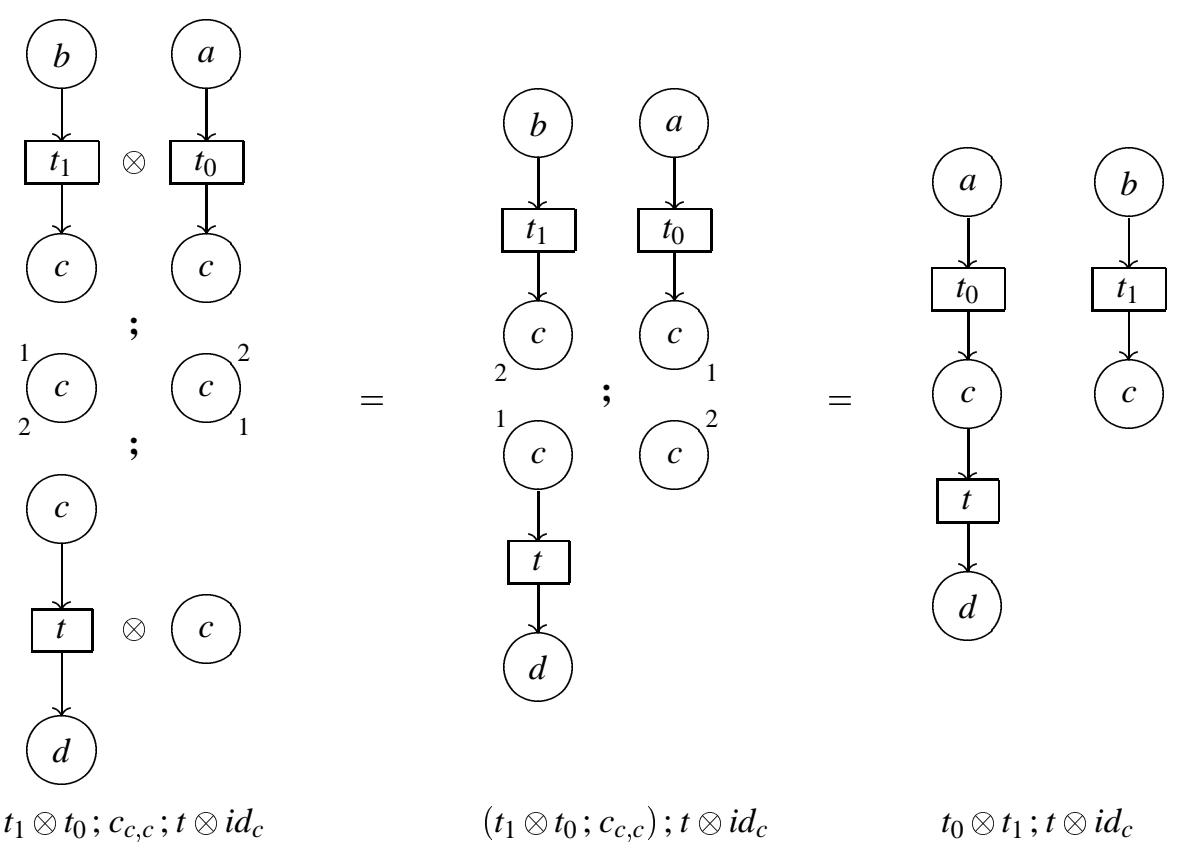

FIGURE 1. A net $N$ and its concatenable process $\pi=t_{0} \otimes t_{1} ; t \otimes i d_{c}$

Observe that, in fact, the strictness of the symmetric structure of the monoidal category has disappeared.

EXAMPLE. Figure 1 shows a concatenable process $\pi$ of a net $N$ that corresponds to the arrow $t_{0} \otimes t_{1} ; t \otimes i d_{c}$ of $\mathcal{P}(N)$. To exemplify the algebra of processes of $N, \pi$ is expressed as parallel $\left({ }_{-} \otimes_{-}\right)$and sequential $\left(_{-} ;_{-}\right)$composition of simpler processes. Such operations are matched precisely by operations and axioms of $\mathcal{P}(N)$, and this is the essence of the theorem above.

The construction $\mathcal{P}\left({ }_{-}\right)$, however, is somehow unsatisfactory, since it is not functorial: given a simulation between two nets, it may not be possible to identify a cor- 


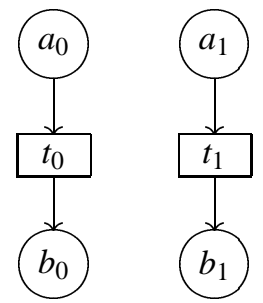

$N$

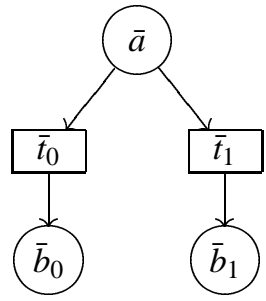

$\bar{N}$

FIGURE 2. The nets $N$ and $\bar{N}$

responding monoidal functor between the respective categories of computations. This fact, besides showing that the understanding of nets provided by $\mathcal{P}($-) is still incomplete, prevents us from identifying the category (of the categories) of net computations, i.e., from axiomatising the behaviour of nets 'in the large'.

DEFINITION. Given nets $N$ and $\bar{N}$, a morphism $f: N \rightarrow \bar{N}$ is a pair $\left\langle f_{T}, f_{S}\right\rangle$, where $f_{T}: T_{N} \rightarrow T_{\bar{N}}$ is a function - the transition component of $f$ - and, denoting by $\mu(S)$ the free commutative monoid on $S, f_{S}: \mu\left(S_{N}\right) \rightarrow \mu\left(S_{\bar{N}}\right)$ is a monoid homomorphism the place component of $f$ - mapping multisets of places of $N$ to multisets of places of $\bar{N}$ in such a way that for all transitions $t: u \rightarrow v$ of $N$, we have $f_{T}(t): f_{S}(u) \rightarrow f_{S}(v)$.

This defines the category Petri of Petri nets.

EXAmPLE. Consider the nets $N$ and $\bar{N}$ in Figure 2 and $f: N \rightarrow \bar{N}$ where $f_{T}\left(t_{i}\right)=\bar{t}_{i}$, $f_{S}\left(a_{i}\right)=\bar{a}$ and $f_{S}\left(b_{i}\right)=\bar{b}_{i}$, for $i=0,1$. Then, $f$ cannot be extended to a monoidal functor $\mathcal{P}(f): \mathcal{P}(N) \rightarrow \mathcal{P}(\bar{N})$. In fact, for any such extension $F$, by monoidality we must have $F\left(t_{0} \otimes t_{1}\right)=F\left(t_{0}\right) \otimes F\left(t_{1}\right)=\bar{t}_{0} \otimes \bar{t}_{1}$, and since $t_{0} \otimes t_{1}=t_{1} \otimes t_{0}$, it follows that

$$
\bar{t}_{0} \otimes \bar{t}_{1}=F\left(t_{1} \otimes t_{0}\right)=\bar{t}_{1} \otimes \bar{t}_{0},
$$

which is impossible, since the leftmost and rightmost processes are different in $\mathcal{P}(\bar{N})$.

\section{Strongly Concatenable Processes}

Strongly concatenable processes are a slight refinement of concatenable processes introduced in $[\mathbf{1 5}, \mathbf{1 7}]$ to yield a functorial algebraic description of net computations. The refinement, which consists simply of decorating selected places in nonsequential processes more strongly than in concatenable processes (see Figure 3), is shown to be - in a very precise mathematical sense (see $[\mathbf{1 5}, \mathbf{1 7}]$ ) — the slightest refinement that yields a functorial construction. As for their predecessors, strongly concatenable processes admit an axiomatisation in terms of a universal algebraic construction based on symmetric monoidal categories.

THEOREM. The strongly concatenable processes of a net $N$ are the arrows of $Q(N)$, obtained from the symmetric strict monoidal category freely generated from the places of $N$ and, for each transition $t$ of $N$, an arrow $t_{u, v}: u \rightarrow v$ for each pair of linearisations (as strings) $u$ and $v$ of the pre-and post- sets (multisets) of $t$, by quotienting modulo the axiom

$$
s ; t_{u^{\prime}, v}=t_{u, v^{\prime}} ; s^{\prime}, \quad \text { for } s: u \rightarrow u^{\prime} \text { and } s^{\prime}: v^{\prime} \rightarrow v \text { symmetries. }
$$




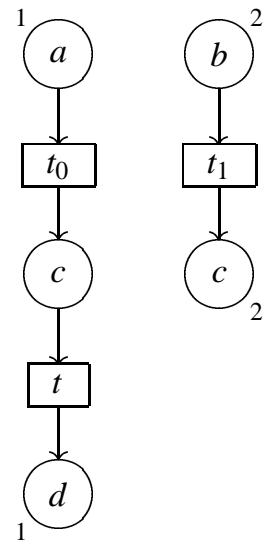

$t_{0} \otimes t_{1} ; t \otimes i d_{c}$

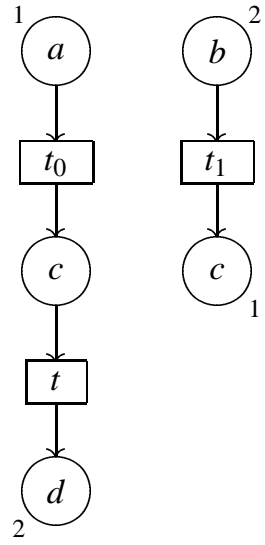

$t_{0} \otimes t_{1} ; c_{c, c} ; i d_{c} \otimes t$

FIGURE 3. Two strongly concatenable processes corresponding to $\pi$ of Figure 1

The key feature of $Q(-)$ is that, differently from $\mathcal{P}(-)$, it associates to the net $N$ a monoidal category whose objects form a free non-commutative monoid (viz. $S_{N}^{*}$ as opposed to $\mu\left(S_{N}\right)$ ), i.e., it deals with strings as explicit representatives of multisets. As hinted above, renouncing to such commutativity, a choice that at first may seem odd, can be proved to be necessary in order to obtain a functor. As a consequence of this choice, each transition of $N$ has many corresponding arrows in $Q(N)$; such arrows, however, are 'related' to each other by the naturality condition $(\Phi)$, in the precise sense that, when collected together, they form a natural transformation between appropriate functors. In fact, $(\Phi)$ asserts that any diagram in $Q(N)$ of the kind

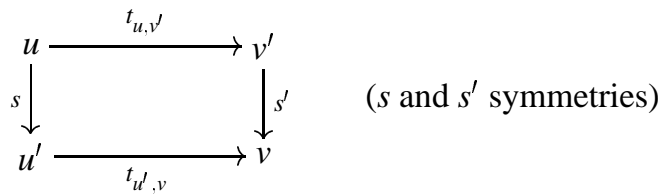

commutes, which, as we shall see, can be equivalently expressed in terms of functors and natural transformations. This naturality axiom is the second relevant feature of $Q(-)$, actually the one that keeps the computational interpretation of the category $Q(N)$, i.e., the strongly concatenable processes, surprisingly close to that of $\mathcal{P}(N)$, i.e., to the concatenable processes (cf. Figures 1 and 3).

Concerning functoriality, [15] shows that $Q($ (-) extends to a coreflection functor from the category of Petri nets to $a$ category of symmetric monoidal categories. The latter is constructed by quotienting the category of symmetric monoidal categories in such a way as to identify all the symmetric monoidal functors that, informally speaking, differ only by the 'representatives of multisets' chosen. Here, we proceed along the way hinted at in [17] using 2-categories (more precisely, groupoid-enriched categories), the role of the 2-structure being to carry information about multisets, thus making an explicit quotient construction unnecessary. 
DEFINITION. A symmetric Petri category is a symmetric strict monoidal category C whose monoid of objects is $S^{*}$, the free monoid on $S$, for some set $S$. We shall let $S_{y} m_{\mathrm{C}}$ denote its subcategory of symmetries, and $\operatorname{Sym}_{\mathrm{C}, \mathrm{V}}$, for $v$ a multiset in $\mu(S)$, the full subcategory of $\operatorname{Sym}_{\mathrm{C}}$ consisting of those $u \in S^{*}$ whose underlying multiset, $\mu(u)$, is $v$.

The relevance of symmetric Petri categories for our purposes resides in that they allow us to capture the essence of the arrows generating $Q(N)$, i.e., the instances of the transitions of $N$. Such arrows, in fact, have two very special properties that characterise them completely: (1) they are decomposable as tensors only trivially, and as compositions only by means of symmetries, and (2), as already mentioned, they form natural transformations between appropriate functors. Following the usual categorical paradigm, we then use such properties, expressed in abstract categorical terms, to define the notion of transition in a general symmetric Petri category.

Definition. Let $C$ be a symmetric Petri category and $S^{*}$ its monoid of objects. An arrow $\tau$ in $C$ is primitive if (denoting by $\varepsilon$ the empty word in $S^{*}$ )

$$
\begin{array}{ll}
\tau & \text { is not a symmetry; } \\
\tau=\alpha ; \beta & \text { implies } \quad \alpha \text { is a symmetry and } \beta \text { is primitive, or viceversa; } \\
\tau=\alpha \otimes \beta & \text { implies } \quad \alpha=i d_{\varepsilon} \text { and } \beta \text { is primitive, or viceversa. }
\end{array}
$$

A transition of $C$ is a natural transformation $\tau: \pi_{C, v, v^{\prime}}^{0} \Rightarrow \pi_{C, v, v^{\prime}}^{1}$ whose components $\tau_{u, v}$ are primitive arrows of $\mathrm{C}$, where, for $\nu, v^{\prime}$ in $\mu(S)$, and the functors $\pi_{C, v, v^{\prime}}^{0}$ and $\pi_{C, v, v^{\prime}}^{1}$ are defined by the diagram

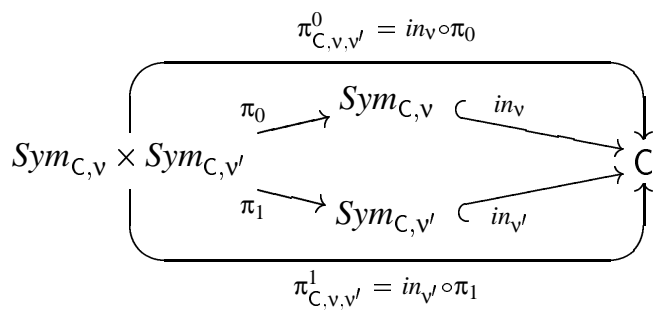

Observe that the definition above captures the essence of $Q(N)$ : the transitions of $Q(N)$ are all and only the families $\left\{t_{u, v} \mid t: \mu(u) \rightarrow \mu(v) \in T_{N}\right\}$. This leads us to the following result.

Theorem. Let SPetriCat be the 2-category whose objects are the symmetric Petri categories, whose arrows are the symmetric strict monoidal functors that respect transitions, and with a 2-cell $F \Rightarrow G$ if there exists a monoidal natural isomorphism between $F$ and $G$ whose components are all symmetries.

Then, $Q\left({ }_{-}\right):$Petri $\rightarrow$ SPetriCat is a pseudo 2-functor (considering the category Petri of Petri nets as a trivial 2-category) that admits a pseudo right adjoint $\mathcal{N}(-)$ forming with $Q(-)$ a pseudo coreflection.

The latter terminology means precisely that the natural family of isomorphisms between homsets that defines an adjunction is relaxed to a (pseudo) natural family of equivalences between homcats. Finally, concerning the category (of the categories) of net computations, we have the following result that characterises abstractly the categories corresponding to algebras of Petri net causal behaviours. 
THEOREM. Let FSPetriCat be the full subcategory of SPetriCat consisting of those symmetric Petri categories $\mathrm{C}$ whose arrows can be generated by tensor and composition from symmetries, and components of transitions of $\mathrm{C}$, uniquely up to the axioms of symmetric strict monoidal categories and the naturality of transitions, i.e., axiom $(\Phi)$.

Then, FSPetriCat and Petri are pseudo equivalent via $\mathcal{N}[-]$ and $Q[-]$.

In the rest of the paper we shall provide a sketch of the proofs of these results.

Let FMon be the category with objects the free monoids $S^{*}$, where $S \in$ Set ranges over sets, and morphisms the monoid homomorphisms. Similarly, let FCMon be the category with objects the free commutative monoids $\mu(S), S \in$ Set, and morphisms the monoid homomorphisms. Consider the obvious quotient functor $(-)^{b}:$ FMon $\rightarrow$ FCMon. Explicitly, $\left(S^{*}\right)^{b}=\mu(S)$, whilst the action of $\left(\_\right)^{b}$ on $f: S^{*} \rightarrow \bar{S}^{*}$ gives the unique homomorphism $f^{b}: \mu(S) \rightarrow \mu(\bar{S})$ such that $f^{b}(a)=\mu(f(a))$ for all $a \in S$. If we regard (_) $)^{b}$ FMon $\rightarrow$ FCMon as a reflexive graph ${ }^{1}$ homomorphism, we can define a reflexive graph homomorphism (_) $)^{\natural}$ : FCMon $\rightarrow$ FMon in the other direction

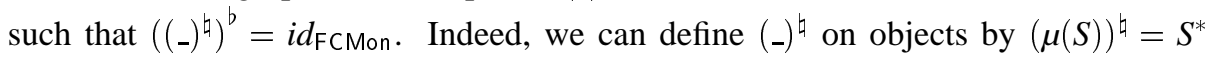
and for each nonidentity monoid homomorphism $f: \mu(S) \rightarrow \mu\left(S^{\prime}\right)$ we can choose a monoid homomorphism $f^{\natural}: S^{*} \rightarrow S^{\prime *}$ by selecting for each $a \in S$ a word $f^{\natural}(a)$ such that $\mu\left(f^{\natural}(a)\right)=f(a)$. For the identity homomorphisms we can define $\left(i d_{\mu(S)}\right)^{\natural}=i d_{S^{*}}$.

Fixed one such (_) $)^{\natural}$, we can define the action of $Q($ (-) on Petri net morphisms.

Let $f: N \rightarrow \bar{N}$ be a morphism of Petri nets. Since $f_{S}$ is a monoid homomorphism from $\mu\left(S_{N}\right)$ to $\mu\left(S_{\bar{N}}\right)$, we consider the homomorphism $f_{S}^{\natural}: S_{N}^{*} \rightarrow S_{\bar{N}}^{*}$. By the freeness of $\operatorname{Sym}_{Q(N)}$, such a morphism can be extended (uniquely) to a symmetric strict monoidal functor $F_{S}: \operatorname{Sym}_{Q(N)} \rightarrow Q(\bar{N})$ and, therefore, to a functor $F: Q(N) \rightarrow Q(\bar{N})$, defined as the unique symmetric strict monoidal functor which coincides with $F_{S}$ on $\operatorname{Sym}_{Q(N)}$ and maps $t_{u, v}: u \rightarrow v$ to $\left(f_{T}(t)\right)_{F(u), F(v)}: F(u) \rightarrow F(v)$. Since monoidal functors map symmetries to symmetries, and since $f_{T}(t)$ is a transition of $N_{1}$, it follows immediately that $F$ respects axiom $(\Phi)$, i.e., that $F$ is well defined. Concerning the (trivial) 2-cells of Petri, the action of $Q(-)$ on them is clearly forced: $Q(i d: f \Rightarrow f)$ must necessarily be (the unique) $Q(f) \Rightarrow Q(f)$.

Since $Q(f)$ is uniquely determined by $f_{T}$ and $f_{S}^{\natural}$, by the property $\left(i d_{\mu(S)}\right)^{\natural}=i d_{S^{*}}$ of ()$\left.^{\natural}\right)^{\natural}$, it follows that $Q\left(i d_{N}\right): Q(N) \rightarrow Q(N)$ is the identity functor. However, since in general $\left(g_{S} \circ f_{S}\right)^{\natural} \neq g_{S}^{\natural} \circ f_{S}^{\natural}$, we have that $Q(g \circ f) \neq Q(g) \circ Q(f)$. The whole point about considering the 2-structure of SPetriCat is, in fact, to show that such functors are isomorphic in SPetriCat, i.e., that $Q[]]$ is a pseudo 2-functor. We proceed as follows.

Let $f: N \rightarrow N^{\prime}$ and $g: N^{\prime} \rightarrow \bar{N}$ be morphisms of nets. Observe that for each $a \in S_{N}$, the string $\left(g_{S} \circ f_{S}\right)^{\natural}(a)$ is a permutation of $g_{S}^{\natural}\left(f_{S}^{\natural}(a)\right)$ and that, therefore, there exists a symmetry $s_{a}: Q(g \circ f)(a) \rightarrow Q(g) \circ Q(f)(a)$ in $Q(\bar{N})$. Then, for $u=u_{1} \cdots u_{n} \in S_{N}^{*}$, take $s_{u}$ to be $s_{u_{1}} \otimes \cdots \otimes s_{u_{n}}: Q(g \circ f)(u) \rightarrow Q(g) \circ Q(f)(u)$. We claim that the family $\left\{s_{u}\right\}_{u \in S_{N}^{*}}$ is a natural transformation $Q(g \circ f) \Rightarrow Q(g) \circ Q(f)$. Since $s$ is clearly a monoidal natural transformation and each $s_{u}$ is a symmetry isomorphism, this proves

\footnotetext{
${ }^{1}$ A reflexive graph has a set $E$ of edges, a set $N$ of nodes, functions $\partial_{0}, \partial_{1}: E \rightarrow N$ and a function $i d: N \rightarrow E$ with $\partial_{i}(i d(x))=x$. Homomorphisms preserve the $\partial_{i}$ and $i d$.
} 


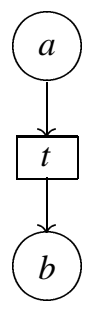

$N$

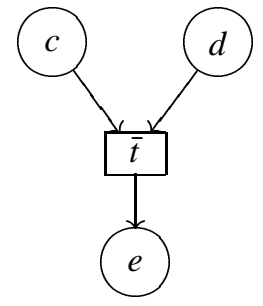

$\bar{N}$

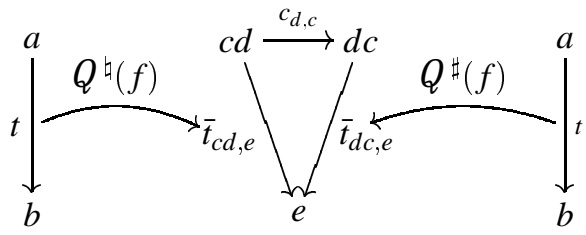

$Q(\bar{N})$

$Q(N)$

FigURE 4. Two different, yet equivalent, pseudo functorial extensions of $Q(-)$

that $Q(g \circ f) \cong Q(g) \circ Q(f)$ in SPetriCat. We proceed by induction on the structure of $\alpha$ to show that, for any $\alpha: u \rightarrow v$ in $Q(N)$, we have

$$
Q(g \circ f)(\alpha) ; s_{v}=s_{u} ; Q(g) \circ Q(f)(\alpha) .
$$

The key to the proof is that $s$ is monoidal, i.e., $s_{u v}=s_{u} \otimes s_{v}$, as a simple inspection of the definition shows. If $\alpha$ is an identity, then the claim is obvious. Moreover, if $\alpha$ is a transition $t_{u, v}$, then $Q(g \circ f)(\alpha)$ and $Q(g) \circ Q(f)(\alpha)$ are instances of the same transition of $\bar{N}$, and the thesis follows immediately from axiom $(\Phi)$. Suppose now that $\alpha=c_{u, v}$, a symmetry of $Q(N)$. Since $Q(g \circ f)$ and $Q(g) \circ Q(f)$ are symmetric strict monoidal functors, the equation that we have to prove reduces to

$$
c_{Q(g \circ f)(u), Q(g \circ f)(v)} ; s_{v} \otimes s_{u}=s_{u} \otimes s_{v} ; c_{Q(g) \circ Q(f)(u), Q(g) \circ Q(f)(v),}
$$

which certainly holds since $\left\{c_{u, v}\right\}_{u, v \in S_{N}^{*}}$ is a natural transformation $x_{1} \otimes x_{2} \Rightarrow x_{2} \otimes x_{1}$. If $\alpha=\alpha_{0} \otimes \alpha_{1}$, with $\alpha_{i}: u_{i} \rightarrow v_{i}$, then, for $i=0,1$, we have by induction hypothesis that $Q(g \circ f)\left(\alpha_{i}\right) ; s_{v_{i}}=s_{u_{i}} ; Q(g) \circ Q(f)\left(\alpha_{i}\right)$ whence

$$
Q(g \circ f)\left(\alpha_{0}\right) \otimes Q(g \circ f)\left(\alpha_{1}\right) ; s_{v_{0} \otimes v_{1}}=s_{u_{0} \otimes u_{1}} ; Q(g) \circ Q(f)\left(\alpha_{0}\right) \otimes Q(g) \circ Q(f)\left(\alpha_{1}\right),
$$

which is $Q(g \circ f)(\alpha) ; s_{v}=s_{u} ; Q(g) \circ Q(f)(\alpha)$. Finally, in the case $\alpha=\alpha_{0} ; \alpha_{1}$, where $\alpha_{0}: u \rightarrow w$ and $\alpha_{1}: w \rightarrow v$, the induction is proved by pasting the two commutative squares in the following diagram, which exists by the induction hypothesis

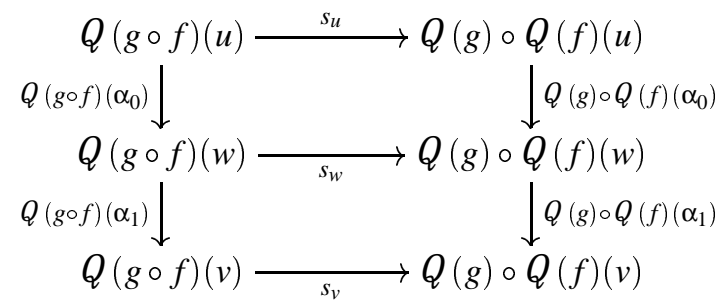

In principle, of course, choosing two different sections for $\left({ }_{-}\right)^{b}$, say (_) $)^{\natural}$ and (_) gives two different pseudo functors $Q^{\natural}\left({ }_{-}\right), Q^{\sharp}(-)$ : Petri $\rightarrow$ SPetriCat (that, however, coincide on the objects). Another use for the 2-structure of SPetriCat is to show that the choice of the 'choice mapping' $\left({ }^{\natural}\right)^{\natural}$ is actually, in a precise mathematical sense, irrelevant. 
EXAMPLE. Let $N$ and $\bar{N}$ be the nets of Figure 4, and let $f: N \rightarrow \bar{N}$ be the morphism such that $f_{T}(t)=\bar{t}, f_{S}(a)=c \oplus d$, and $f_{S}(b)=e$. In the hypothesis that $f^{\natural}(a)=c d$ and $f^{\sharp}(a)=d c$, we have that $Q^{\natural}(f)\left(t_{a, b}\right)=\bar{t}_{c d, e} \neq \bar{t}_{d c, e}=Q^{\sharp}(f)\left(t_{a, b}\right)$.

Observe, however, that $\bar{t}_{c d, e}$ and $\bar{t}_{d c, e}$ are simply different instances of the same transition of $\bar{N}$, namely $\bar{t}: c \oplus d \rightarrow e$, related by axiom $(\Phi)$ via the symmetries $c_{c, d}$ and $i d_{e}$. In other words, $\bar{t}_{c d, e}$ and $\bar{t}_{d c, e}$ are components of the same transition of $Q(N)$. This fact holds in full generality: for each net morphism $f, Q^{\natural}(f)$ and $Q^{\sharp}(f)$ differ at most by the correspondence between components of transitions, and coincide when considered as maps of transitions of $Q(N)$ to transitions of $Q(\bar{N})$ (cf. $[15,17]$ ); formally, there exists a 2-cell $Q^{\natural}(f) \Rightarrow Q^{\sharp}(f)$ in SPetriCat that makes them isomorphic. In this sense, $Q^{\natural}(-)$ and $Q^{\sharp}(-)$ are equivalent functors, and the choice of the section of $\left(_{-}\right)^{b}$ is irrelevant.

In order to make this precise, let ()$^{\natural}$ and $\left(_{-}\right)^{\sharp}$ be sections of $\left(_{-}\right)^{b}$ and let $f: N \rightarrow \bar{N}$ be a morphism of nets. Since for each $f: \mu(S) \rightarrow \mu\left(S^{\prime}\right)$ any such section can only differ in the choice of the linearization of each multiset $f(a), a \in S$, the string $f^{\natural}(a)$ is a permutation of $f^{\sharp}(a)$ for each $a \in S_{N}$. Then, we can choose a symmetry $s_{a}: Q^{\natural}(f)(a) \rightarrow$ $Q^{\sharp}(f)(a)$ in $Q(\bar{N})$, take $s_{u_{1}} \otimes \cdots \otimes s_{u_{n}}: Q^{\natural}(f)(u) \rightarrow Q^{\sharp}(f)(u)$ as $s_{u}$ for each $u=$ $u_{1} \cdots u_{n} \in S_{N}^{*}$, and - proceeding as before - prove that $s=\left\{s_{u}\right\}_{u \in S_{N}^{*}}$ is a natural transformation $Q^{\natural}(f) \Rightarrow Q^{\sharp}(f)$. Since $s$ is monoidal and each $s_{u}$ is a symmetry isomorphism, this proves that $Q^{\natural}(f) \cong Q^{\sharp}(f)$ in SPetriCat.

Let us now turn our attention to $\mathcal{N}(-)$. There is now an obvious way to extract a net $\mathcal{N}(\mathrm{C})$ from a symmetric Petri category C, namely:

- the places of $\mathcal{N}(\mathrm{C})$ are the generators $S$ of the monoid of objects $S^{*}$ of C;

- a transition $\tau: v \rightarrow v^{\prime}$ of $\mathcal{N}(C)$ is a transition $\tau: \pi_{C, v, v^{\prime}}^{0} \Rightarrow \pi_{C, v, v^{\prime}}^{1}$ of $C$.

Let $F: \mathrm{C} \rightarrow \overline{\mathrm{C}}$ be a morphism of symmetric Petri categories, and let $S^{*}$ and $\bar{S}^{*}$ be the monoid of objects of, respectively, $\mathrm{C}$ and $\overline{\mathrm{C}}$. The object component of $F$, say $F_{S}$, induces a monoid homomorphism $F_{S}^{b}: \mu(S) \rightarrow \mu(\bar{S})$. Moreover, since $F$ respects transitions, i.e., since for each transition $\tau$ of $C$ there exists a (necessarily unique) transition $\bar{\tau}$ of $\bar{C}$ such that $F\left(\tau_{u, v}\right)=\bar{\tau}_{F(u), F(v)}$, its arrow component restricts to a map $F_{T}$ from the transitions of $\mathcal{C}$ to those of $\bar{C}$ in such a way that, if $\tau: v \rightarrow v^{\prime}$ in $\mathcal{N}(C)$, then $F_{T}(\tau): F_{S}^{b}(v) \rightarrow F_{S}^{b}\left(v^{\prime}\right)$ in $\mathcal{N}(\bar{C})$. Therefore, $\left\langle F_{T}, F_{S}\right\rangle: \mathcal{N}(\mathrm{C}) \rightarrow \mathcal{N}(\overline{\mathrm{C}})$ is a morphism of nets, which we will denote $\mathcal{N}(F)$. It follows easily from the functoriality of (_) that $\mathcal{N}(-)$, as defined above, is a (1-)functor. Concerning 2-cells, it can be proved that whenever $F \Rightarrow G$ in SPetriCat, i.e., $F$ and $G$ are isomorphic via a monoidal natural transformation composed exclusively of symmetries, then $F_{S}^{b}=G_{S}^{b}$ and $F_{T}=G_{T}$, which means that $\mathcal{N}(F)=\mathcal{N}(G)$. Therefore, defining $\mathcal{N}(F \Rightarrow G)=i d_{\mathfrak{N}(F)}$ yields a 2-functor $\mathcal{N}\left({ }_{-}\right)$: SPetriCat $\rightarrow$ Petri.

The last argument shows that $\mathcal{N}(-)$, although full, is not faithful. Observe now that $N$ and $\mathcal{N}(Q(N))$ are isomorphic via the Petri net morphism $\eta_{N}: N \rightarrow \mathcal{N}(Q(N))$ whose place component is $i d_{S_{N}}$ and whose transition component maps $t \in T_{N}$ to the transition $\left\{t_{u, v} \in Q(N)\right\} \in T_{\mathcal{N}(Q(N))}$. These two facts imply that $\mathcal{N}\left({ }_{-}\right)$cannot be adjoint to $Q\left({ }_{-}\right)$, as the homcats SPetriCat $(Q(N), \mathrm{C})$ and $\operatorname{Petri}(N, \mathcal{N}(\mathrm{C})) \cong \operatorname{Petri}(\mathcal{N}(Q(N)), \mathcal{N}(\mathrm{C}))$ are not isomorphic. Actually, since $\mathcal{N}(-)$ performs a quotient of monoids, viz. (-) $)^{b}$, 
whilst $Q(-)$ chooses an arbitrary linearisation for the place components of net morphisms, viz. (_) $)^{\natural}$, one could not expect otherwise. We shall see next that $\mathcal{N}\left({ }_{-}\right)$identifies only isomorphic functors or, equivalently, that the categories SPetriCat $(Q(N), \mathrm{C})$ and Petri $(N, \mathcal{N}(\mathrm{C}))$ are equivalent.

Let $C$ be a symmetric Petri category $C$, let $\varepsilon_{C}: Q \mathcal{N}(C) \rightarrow C$ be the (unique) symmetric strict monoidal functor that acts identitically on objects and sends the component at $(u, v)$ of the transition $\tau: v \rightarrow v^{\prime}$ of $\mathcal{L}(\mathrm{C})$ to the component $\tau_{u, v}$ of the corresponding natural transformation $\tau: \pi_{C, v, v^{\prime}}^{0} \Rightarrow \pi_{C, v, v^{\prime}}^{1}$ of $C$. Since $\tau$ is a transition of $C, \varepsilon_{C}$ is well defined. Since it preserves transitions, it is a morphism of symmetric Petri categories.

Since $\mathcal{N}(-)$ is a 2-functor and $Q(-)$ acts functorially on 2-cells, for each $N$ in Petri and each $C$ in SPetriCat, the functors $\eta_{N}$ and $\varepsilon_{C}$ induce a pair of functors between homcats as follows.

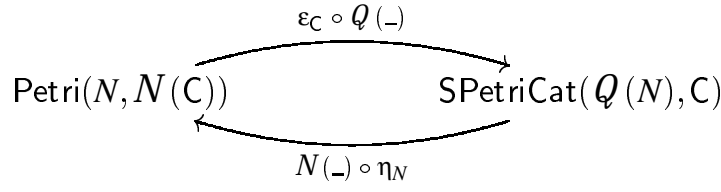

Let us prove that such functors form an equivalence of categories.

For $f: N \rightarrow \mathcal{N}(C)$, let $g$ be $\mathcal{N}\left(\varepsilon_{C} \circ Q(f)\right) \circ \eta_{N}$. Since $g(a)=\left(f^{\natural}\right)^{b}(a)=f(a)$ and $g(t)=\mathcal{N}\left(\varepsilon_{\mathrm{C}} \circ Q(f)\right)\left(\left\{t_{u, v}\right\}\right)=f(t)$, we have that $g=f$. In the opposite direction, for $F: Q(N) \rightarrow C$, let $G$ stand for $\varepsilon_{C} \circ Q\left(\mathcal{N}(F) \circ \eta_{N}\right)$, and consider $a \in S_{N}$. Since $\mathcal{N}(F) \circ \eta_{N}(a)=F_{S}^{\mathrm{b}}(a)$, we have that $G(a)=Q\left(\mathcal{N}(F) \circ \eta_{N}(a)\right)=\left(F_{S}^{b}\right)^{\natural}(a)$ and, thus, there exists a symmetry $s_{a}: F(a) \rightarrow G(a)$ in $C$. Then, for $u=u_{1} \cdots u_{n} \in S_{N}^{*}$, let $s_{u}$ be $s_{u_{1}} \otimes \cdots \otimes s_{u_{n}}: F(u) \rightarrow G(u)$. An inductive argument completely analogous to the one exploited previously shows that the family $s=\left\{s_{u}\right\}_{u \in S_{N}^{*}}$ is a monoidal natural isomorphism $F \Rightarrow G$ whose components are all symmetries. Therefore $F \cong G$ in SPetriCat, completing the proof that $\mathcal{N}\left(\varepsilon_{\mathrm{C}} \circ Q(-)\right) \circ \eta_{N}=i d$ and $\varepsilon_{C} \circ Q\left(\mathcal{N}\left({ }_{-}\right) \circ \eta_{N}\right) \cong i d$, i.e., that $\varepsilon_{C} \circ Q(-)$ and $\mathcal{N}\left({ }_{-}\right) \circ \eta_{N}$ form an equivalence of categories.

To show that $Q\left({ }_{-}\right) \dashv \mathcal{N}\left(\left(_{-}\right)\right.$, i.e., that $\mathcal{N}\left({ }_{-}\right)$is pseudo right adjoint to $Q\left({ }_{-}\right)$, we need to show that the above family of equivalences is pseudo natural - that is natural up to isomorphism - in N and C. This amounts to showing that, for each $g: \bar{N} \rightarrow N$ and $G: \mathrm{C} \rightarrow \overline{\mathrm{C}}$, the following diagrams of functors commute up to isomorphism.

$$
\begin{gathered}
\operatorname{Petri}(N, \mathcal{N}(\mathrm{C})) \stackrel{\varepsilon_{\mathrm{C}} \circ Q(-)}{\longrightarrow} \operatorname{SPetriCat}(Q(N), \mathrm{C}) \stackrel{\mathcal{N}(-) \circ \eta_{N}}{\longrightarrow} \operatorname{Petri}(N, \mathcal{N}(\mathrm{C})) \\
\mathcal{N}(G) \circ-\circ g \downarrow \\
\operatorname{Petri}(\bar{N}, \mathcal{N}(\overline{\mathrm{C}})) \underset{\varepsilon_{\overline{\mathrm{C}}} \circ Q(-)}{\longrightarrow} \operatorname{SPetriCat}(Q(\bar{N}), \overline{\mathrm{C}}) \underset{\mathcal{N}(-) \circ \eta_{\bar{N}}}{\longrightarrow} \operatorname{Petri}(\bar{N}, \mathcal{N}(\overline{\mathrm{C}}))
\end{gathered}
$$

It is in fact routine to prove that $G \circ \varepsilon_{\mathrm{C}} \circ Q(-\circ g)=\varepsilon_{\overline{\mathrm{C}}} \circ Q\left(\mathcal{N}(G) \circ \_\circ g\right)$ and that $\mathcal{N}\left(G \circ \circ_{-}\right) \circ \eta_{N} \circ g \cong \mathcal{N}\left(G \circ \circ_{-} \circ Q(g)\right) \circ \eta_{\bar{N}}$.

Finally, concerning FSPetriCat, by general arguments in category theory, it is enough to show that $C$ belongs to FSPetriCat if and only if $\varepsilon_{\mathrm{C}}: Q \mathcal{N}(\mathrm{C}) \rightarrow \mathrm{C}$, the component at $C$ of the counit of $Q(-) \dashv \mathcal{N}\left(\left(_{-}\right)\right.$is an iso. Since $\varepsilon_{C}$ is an isomorphism on the objects, it is iso if and only if it is an isomorphism on each homset. Then the result follows, since each arrow of $C$ can be written as tensor and composition of symmetries 
and component of transitions if and only if $\varepsilon_{\mathrm{C}}$ is surjective on each homset, and this can be done uniquely (up to the equalities that necessarily hold in any symmetric Petri category) if and only if $\varepsilon_{C}$ is injective on each homset.

\section{References}

[1] E. Best and R. Devillers (1987), Sequential and Concurrent Behaviour in Petri Net Theory, Theoretical Computer Science n. 55, $87-136$.

[2] C. Brown, D. Gurr, ANd V. DE PAIVA (1991), A Linear Specification Language for Petri Nets, Technical Report DAIMI PB-363, Computer Science Department, University of Aarhus.

[3] P. Degano, J. Meseguer, And U. Montanari (1989), Axiomatizing Net Computations and Processes, in Proceedings LICS'89, IEEE Computer Society Press, $175^{-18}$.

[4] P. Degano, J. Meseguer, And U. Montanari (1996), Axiomatizing the Algebra of Net Computations and Processes, in Acta Informatica n. 33, 641-667.

[5] U. Goltz And W. Reisig (1983), The Non Sequential Behaviour of Petri Nets, Information and Computation n. 57, $125^{-147}$.

[6] J. Meseguer and U. Montanari (1990), Petri Nets are Monoids, Information and Computation n. $88,105^{-1} 54$.

[7] J. Meseguer, U. Montanari, and V. Sassone (1996), Process versus Unfolding Semantics for Place/Transition Petri Nets, Theoretical Computer Science n. 153, 171-210.

[8] J. Meseguer, U. Montanari, and V. Sassone (1997), On the Semantics of PlaceTransition Petri Nets, Mathematical Structures in Computer Science, to appear. Avalaible by anonymous ftp at http://www.di.unipi.it/ vs.

[9] M. Nielsen, G. Plotkin, AND G. Winskel (1981), Petri Nets, Event Structures and Domains, Part 1, Theoretical Computer Science n. 13, 85-108.

[10] C.A. Petri (1962), Kommunikation mit Automaten. PhD thesis, Institut für Instrumentelle Mathematik.

[11] C.A. PETRI (1973), Concepts of Net Theory, in Proceedings of MFCS'73, Mathematics Institute of the Slovak Academy of Science, 137-146.

[12] C.A. Petri (1977), Non Sequential Processes, Interner Bericht ISF-77-5, Gesellschaft für Mathematik und Datenverarbeitung.

[13] W. ReISIG (1985), Petri Nets. Springer-Verlag.

[14] V. Sassone (1995), Axiomatizing Petri Net Concatenable Processes, in Proceedings of FCT'95, H. Reichel (Ed.), LNCS n. 965, Springer-Verlag, 414-423.

[15] V. SAssone (1995), On the Category of Petri Net Computations, in Proceedings of TAPSOFT'95, P.D. Mosses et al (Eds.), LNCS n. 915, Springer-Verlag, 334-348.

[16] V. SAssone (1996), An Axiomatization of the Algebra of Petri Net Concatenable Processes, Theoretical Computer Science n. 1 70, 277-296.

[17] V. Sassone, An Axiomatization of the Category of Petri Net Computations. Submitted for publication. Avalaible by anonymous ftp at http://www.di.unipi.it/ vs.

[18] G. Winskel (1987), Petri Nets, Algebras, Morphisms and Compositionality, Information and Computation n. $\mathbf{7 2}^{2}, 197^{-23} 8$.

Computer Science Lab., SRi International, 333 Ravenswood Ave., Menlo Park, CA

E-mail address: meseguer@csl.sri.com

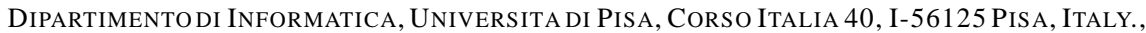
ON LEAVE DURING 1996-97 AT COMPUTER SCIENCE LAB., SRI INTERNATIONAL.

E-mail address: ugo@di.unipi.it

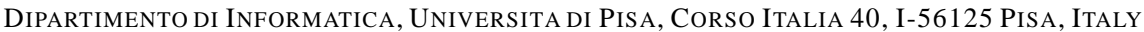

E-mail address: vs@di.unipi.it 